\title{
Factual Power Loss Diminution by Enriched Artificial Fish Swarm Algorithm
}

\author{
Kanagasabai Lenin \\ Department of EEE, Prasad V. Potluri Siddhartha Institute of Technology, Kanuru, Vijayawada, Andhra Pradesh -520007, India.
}

How to cite this paper: Kanagasabai Lenin. (2020) Factual Power Loss Diminution by Enriched Artificial Fish Swarm Algorithm. Journal of Electrical Power \& Energy Systems, 4(1), 1-10.

DOI: $10.26855 /$ jepes.2020.05.001

Received: March 22, 2020

Accepted: April 30, 2020

Published: May 8, 2020

*Corresponding author: Kanagasabai Lenin, Department of EEE, Prasad V.

Potluri Siddhartha Institute of

Technology, Kanuru, Vijayawada,

Andhra Pradesh -520007, India.

Email: gklenin@gmail.com

\begin{abstract}
In this work Enriched Artificial Fish swarm (EAFS) algorithm is projected to solve optimal reactive power problem. In the proposed algorithm, food concentration function, bulletin board approach, target position search mechanism, and position move method are utilized. Subsequently, an adjustment strategy of exploration range of artificial fish, which merge the global search with local search, is projected to enhance the explore capability of the projected algorithm. Every artificial fish will execute the swarming behavior, following behavior and foraging behavior in order to discover the goal move position $X_{i}^{n e x t}$ with the superior food concentration. The position with the uppermost food concentration of the new-fangled positions (Xnext1, Xnext2 andXnext3) are found by these behaviours' is used as $X_{i}^{\text {next }}$. Proposed Enriched Artificial Fish swarm (EAFS) algorithm has been tested in standard IEEE $14,30,57,118,300$ bus test systems and simulation results show the projected algorithm reduced the real power loss comprehensively.
\end{abstract}

\section{Keywords}

Optimal Reactive Power, Transmission Loss, Enriched Artificial Fish Swarm Algorithm

\section{Introduction}

Reactive power problem plays an important role in secure and economic operations of power system. Numerous types of methods [1-6] have been utilized to solve the optimal reactive power problem. However many scientific difficulties are found while solving problem due to an assortment of constraints. Evolutionary techniques [7-16] are applied to solve the reactive power problem. This paper proposes Enriched Artificial Fish swarm (EAFS) algorithm to solve optimal reactive power problem. Artificial fish swarm algorithm inspired from the natural behaviours' of fish swarm searching food in water, the artificial fish swarm algorithm. In the proposed Enriched Artificial Fish swarm (EAFS) algorithm which uses the adjustment strategy of exploration range of the artificial fish in iterations. Bulletin board is utilized for recording the optimal position with the utmost food concentration which has been found by fish swarm. In initial iteration, the bulletin board will record the optimal position of the preliminary positions of artificial fish. When the artificial fish moves to a new-fangled position for finding food with superior food concentration, the bulletin board is modernized to record the optimal position discovered so far. After finding $X_{i}^{\text {next }}$, the ith artificial fish make a decision about the movement to $X_{i}^{\text {next }}$. When the food concentration of $X_{i}^{\text {next }}$ is superior to $X_{\mathrm{i}}$, then the ith artificial fish move towards to $X_{i}^{\text {next }}$ from 
$\mathrm{X}_{\mathrm{i}}$, then $\mathrm{X}_{\mathrm{i}}$ is modernized by $X_{i}^{\text {next }}$. Or else, ith artificial fish gives up moving towards $X_{i}^{\text {next }}$, then $\mathrm{X}_{\mathrm{i}}$ stay unmoved. Proposed Enriched Artificial Fish Swarm (EAFS) algorithm has been tested in standard IEEE 14, 30, $57,118,300$ bus test systems and simulation results show the projected algorithm reduced the real power loss comprehensively.

\section{Problem Formulation}

Objective of the problem is to reduce the true power loss:

$$
\mathrm{F}=\mathrm{P}_{\mathrm{L}}=\sum_{\mathrm{k} \in \mathrm{Nbr}} \mathrm{g}_{\mathrm{k}}\left(\mathrm{V}_{\mathrm{i}}^{2}+\mathrm{V}_{\mathrm{j}}^{2}-2 \mathrm{~V}_{\mathrm{i}} \mathrm{V}_{\mathrm{j}} \cos \theta_{\mathrm{ij}}\right)
$$

Voltage deviation given as follows:

$$
\mathrm{F}=\mathrm{P}_{\mathrm{L}}+\omega_{\mathrm{v}} \times \text { Voltage Deviation }
$$

Voltage deviation given by:

$$
\text { Voltage Deviation } \quad=\sum_{\mathrm{i}=1}^{\mathrm{Npq}}\left|\mathrm{V}_{\mathrm{i}}-1\right|
$$

Constraint (Equality)

$$
\mathrm{P}_{\mathrm{G}}=\mathrm{P}_{\mathrm{D}}+\mathrm{P}_{\mathrm{L}}
$$

Constraints (Inequality)

$$
\begin{aligned}
& \mathrm{P}_{\text {gslack }}^{\min } \leq \mathrm{P}_{\text {gslack }} \leq \mathrm{P}_{\text {gslack }}^{\max } \\
& \mathrm{Q}_{\mathrm{gi}}^{\text {min }} \leq \mathrm{Q}_{\mathrm{gi}} \leq \mathrm{Q}_{\mathrm{gi}}^{\text {max }}, \mathrm{i} \in \mathrm{N}_{\mathrm{g}} \\
& \mathrm{V}_{\mathrm{i}}^{\min } \leq \mathrm{V}_{\mathrm{i}} \leq \mathrm{V}_{\mathrm{i}}^{\max }, \mathrm{i} \in N \\
& \mathrm{~T}_{\mathrm{i}}^{\min } \leq \mathrm{T}_{\mathrm{i}} \leq \mathrm{T}_{\mathrm{i}}^{\max }, \mathrm{i} \in \mathrm{N}_{\mathrm{T}} \\
& \mathrm{Q}_{\mathrm{c}}^{\min } \leq \mathrm{Q}_{\mathrm{c}} \leq \mathrm{Q}_{\mathrm{C}}^{\max }, \mathrm{i} \in \mathrm{N}_{\mathrm{C}}
\end{aligned}
$$

\section{Enriched Artificial Fish Swarm Algorithm}

Artificial fish swarm algorithm inspired from the natural behaviors of fish swarm searching food in water, the artificial fish swarm algorithm. In the proposed Enriched Artificial Fish swarm (EAFS) algorithm which uses the adjustment strategy of exploration range of the artificial fish in iterations.

The feasible preliminary positions of "N" artificial fish in the fish swarm are arbitrarily engendered to wrap the whole exploration space,

$$
X_{i}=\left[x_{(i, 1)}, x_{(i, 2),} x_{(i, j)}, . ., x_{(i, n)}\right]
$$

$x_{(i, j)}$ has to satisfy the predefined range,

$$
x_{\min } \leq x_{(i, j)} \leq x_{\max }
$$

Food concentration of the location of the artificial fish is utilized for assessing the capability of the solution to solve the reactive power problem,

$$
F c=F(o b j, p t y)
$$

Obj ; objective function value of the position, and $\mathrm{Fc}$; food concentration of the position

Bulletin board is utilized for recording the optimal position with the utmost food concentration which has been found by fish swarm. In initial iteration, the bulletin board will record the optimal position of the preliminary positions of artificial fish. When the artificial fish moves to a new-fangled position for finding food with superior food concentration, the bulletin board is modernized to record the optimal position discovered so far.

Every artificial fish will execute the swarming behavior, following behavior and foraging behavior in order to discover the goal move position $X_{i}^{\text {next }}$ with the superior food concentration. The position with the uppermost food concentration of the new-fangled positions (Xnext1, Xnext2 andXnext3) are found by these behaviors is used as $X_{i}^{\text {next }}$.

Swarming Behaviour 
Assume that the present state of artificial fish is $\mathrm{X}_{\mathrm{i}}\left(\mathrm{d}_{\mathrm{i} . \mathrm{j}}<\right.$ Visual $)$, number of artificial fish is $\mathrm{n}_{\mathrm{f}}$, if $\mathrm{n}_{\mathrm{f}}<\delta$ indicate that the cohort [17] have more food and less crowded, if $X_{c}$ better than $X_{i}$,subsequently move towards the center of the direction of the partnership,

$$
X n e x t 1=X_{i}+\text { random }(0,1) * \operatorname{step}_{\text {iteration }} * \frac{X_{c}-X_{i}}{\left\|X_{c}-X_{i}\right\|}
$$

Following Behaviour

Every artificial fish discover the most excellent position VBX with the uppermost food concentration in its visual range,

$$
X n e x t 2=X_{i}+\operatorname{random}(0,1) * \operatorname{step}_{\text {iteration }} * \frac{V B X-X_{i}}{\left\|V B X-X_{i}\right\|}
$$

Foraging behavior

Choose the arbitrary position $\mathrm{T} X$ in the visual range of the ith artificial fish by using Equation below,

$$
\begin{aligned}
& T X=X_{i}+\text { visual }_{\text {iteration }} *(-1+2 * \text { random }[1, n]) \\
& X \text { next3 }=X_{i}+\text { random }(0,1) * \text { step }_{\text {iteration }} * \frac{T X-X_{i}}{\left\|T X-X_{i}\right\|}
\end{aligned}
$$

Random behavior

The random behavior is implemented by the ith artificial fish, when swarming behavior, following behavior and foraging behavior is not successful,

$$
X \text { next } 4=X_{i}+\text { step }_{\text {iteration }} *(-1+2 * \operatorname{random}[1, n])
$$

Position move

After finding $X_{i}^{\text {next }}$, the ith artificial fish make a decision about the movement to $X_{i}^{\text {next }}$. When the food concentration of $X_{i}^{\text {next }}$ is superior to $\mathrm{X}_{\mathrm{i}}$, then the ith artificial fish move towards to $X_{i}^{\text {next }}$ from $\mathrm{X}_{\mathrm{i}}$, then $\mathrm{X}_{\mathrm{i}}$ is modernized by $X_{i}^{\text {next }}$. Or else, ith artificial fish gives up moving towards $X_{i}^{\text {next }}$, then $\mathrm{X}_{\mathrm{i}}$ stay unmoved.

The values of visual iteration $_{\text {and }}$ step $_{\text {iteration }}$ of artificial fish are animatedly attuned in the every iteration. The visual $_{\text {iteration }}$ and step $_{\text {iteration }}$ computed by,

$$
\begin{aligned}
& \text { visual }_{\text {iteration }}=\frac{(\text { maximum number of iteration-current iteration })^{t}}{(\text { maximum number of iteration })^{t}} *(\text { Vin }-V f n)+V f n \\
& \text { step }_{\text {iteration }}=\frac{(\text { maximum number of iteration-current iteration })^{t}}{(\text { maximum number of iteration })^{t}} *(S i n-S f n)+S f n
\end{aligned}
$$

\footnotetext{
a. Begin

b. Position of the "N" artificial fish is initialized the in fish swarm

c. Iter=1

d. Bulletin board is initialized

e. Compute the values of visual iteration $_{\text {and }}$ step $_{\text {iteration }}$
}

f. Every artificial fish will execute the swarming behavior, following behavior and foraging behavior in order to discover the goal move position $X_{i}^{\text {next }}$ with the superior food concentration.

g. After finding $X_{i}^{\text {next }}$, the ith artificial fish make a decision about the movement to $X_{i}^{\text {next }}$. When the food concentration of $X_{i}^{\text {next }}$ is superior to $\mathrm{X}_{\mathrm{i}}$, then the ith artificial fish move towards to $X_{i}^{\text {next }}$ from $\mathrm{X}_{\mathrm{i}}$, then $\mathrm{X}_{\mathrm{i}}$ is modernized by $X_{i}^{\text {next }}$. Or else, ith artificial fish gives up moving towards $X_{i}^{\text {next }}$, then $\mathrm{X}_{\mathrm{i}}$ stay unmoved.

h. Bulletin board updated

i. $\quad$ Iter $=$ iter +1

j. $\quad$ iter $>$ maximum number of iteration if yes stop and output the result or else go to step e

\section{Simulation Results}

At first in standard IEEE 14 bus system the validity of the proposed Enriched Artificial Fish swarm (EAFS) algorithm has been tested, Table 1 shows the constraints of control variables Table 2 shows the limits of reactive power generators and comparison results are presented in Table 3. 
Table 1. Constraints of control variables

\begin{tabular}{cccc}
\hline System & Variables & Minimum (PU) & Maximum (PU) \\
\hline \multirow{2}{*}{ IEEE 14 Bus } & Generator Voltage & 0.95 & 1.1 \\
& Transformer Tap & 0.9 & 1.1 \\
& VAR Source & 0 & 0.20 \\
\hline
\end{tabular}

Table 2. Constrains of reactive power generators

\begin{tabular}{|c|c|c|c|}
\hline System & Variables & Q Minimum (PU) & Q Maximum (PU) \\
\hline & 1 & 0 & 10 \\
\hline \multirow[t]{4}{*}{ IEEE 14 Bus } & 2 & -40 & 50 \\
\hline & 3 & 0 & 40 \\
\hline & 6 & -6 & 24 \\
\hline & 8 & -6 & 24 \\
\hline
\end{tabular}

Table 3. Simulation results of IEEE - 14 system

\begin{tabular}{ccccccc}
\hline Control variables & Base case & MPSO [19] & PSO [19] & EP [19] & SARGA [19] & EAFS \\
\hline$V G-1$ & 1.060 & 1.100 & 1.100 & $\mathrm{NR}^{*}$ & $\mathrm{NR}^{*}$ & 1.028 \\
$V G-2$ & 1.045 & 1.085 & 1.086 & 1.029 & 1.060 & 1.032 \\
$V G-3$ & 1.010 & 1.055 & 1.056 & 1.016 & 1.036 & 1.029 \\
$V G-6$ & 1.070 & 1.069 & 1.067 & 1.097 & 1.099 & 1.030 \\
$V G-8$ & 1.090 & 1.074 & 1.060 & 1.053 & 1.078 & 1.013 \\
Tap 8 & 0.978 & 1.018 & 1.019 & 1.04 & 0.95 & 0.907 \\
Tap 9 & 0.969 & 0.975 & 0.988 & 0.94 & 0.95 & 0.929 \\
Tap 10 & 0.932 & 1.024 & 1.008 & 1.03 & 0.96 & 0.940 \\
$Q C-9$ & 0.19 & 14.64 & 0.185 & 0.18 & 0.06 & 0.151 \\
$P G$ & 272.39 & 271.32 & 271.32 & $\mathrm{NR} *$ & $\mathrm{NR} *$ & 271.27 \\
$Q G$ (Mvar) & 82.44 & 75.79 & 76.79 & $\mathrm{NR} *$ & $\mathrm{NR} *$ & 74.92 \\
Reduction in PLoss (\%) & 0 & 9.2 & 9.1 & 1.5 & 2.5 & 12.37 \\
Total PLoss (Mw) & 13.550 & 12.293 & 12.315 & 13.346 & 13.216 & 11.873 \\
\hline
\end{tabular}

NR* - Not reported.

Then the proposed Enriched Artificial Fish swarm (EAFS) algorithm has been tested, in IEEE 30 Bus system. Table 4 shows the constraints of control variables, Table 5 shows the limits of reactive power generators and comparison results are presented in Table 6 .

Table 4. Constraints of control variables

\begin{tabular}{cccc}
\hline \multirow{2}{*}{ System } & Variables & Minimum (PU) & Maximum (PU) \\
\hline \multirow{2}{*}{ IEEE 30 Bus } & Generator Voltage & 0.95 & 1.1 \\
& Transformer Tap & 0.9 & 1.1 \\
& VAR Source & 0 & 0.20 \\
\hline
\end{tabular}


Table 5. Constrains of reactive power generators

\begin{tabular}{cccc}
\hline System & Variables & Q Minimum (PU) & Q Maximum (PU) \\
\hline \multirow{2}{*}{ IEEE 30 Bus } & 1 & 0 & 50 \\
& 2 & -40 & 40 \\
& 5 & -40 & 40 \\
& 8 & -10 & 24 \\
& 11 & -6 & 24 \\
\hline
\end{tabular}

Table 6. Simulation results of IEEE -30 system

\begin{tabular}{|c|c|c|c|c|c|c|}
\hline Control variables & Base case & MPSO [19] & PSO [19] & EP [19] & SARGA [19] & EAFS \\
\hline$V G-1$ & 1.060 & 1.101 & 1.100 & $\mathrm{NR}^{*}$ & $\mathrm{NR}^{*}$ & 1.039 \\
\hline$V G-2$ & 1.045 & 1.086 & 1.072 & 1.097 & 1.094 & 1.026 \\
\hline$V G-5$ & 1.010 & 1.047 & 1.038 & 1.049 & 1.053 & 1.057 \\
\hline$V G-8$ & 1.010 & 1.057 & 1.048 & 1.033 & 1.059 & 1.028 \\
\hline$V G-12$ & 1.082 & 1.048 & 1.058 & 1.092 & 1.099 & 1.063 \\
\hline VG-13 & 1.071 & 1.068 & 1.080 & 1.091 & 1.099 & 1.056 \\
\hline Tap11 & 0.978 & 0.983 & 0.987 & 1.01 & 0.99 & 0.912 \\
\hline Tap12 & 0.969 & 1.023 & 1.015 & 1.03 & 1.03 & 0.928 \\
\hline Tap15 & 0.932 & 1.020 & 1.020 & 1.07 & 0.98 & 0.912 \\
\hline Tap36 & 0.968 & 0.988 & 1.012 & 0.99 & 0.96 & 0.919 \\
\hline QC10 & 0.19 & 0.077 & 0.077 & 0.19 & 0.19 & 0.098 \\
\hline QC24 & 0.043 & 0.119 & 0.128 & 0.04 & 0.04 & 0.126 \\
\hline$P G(\mathrm{MW})$ & 300.9 & 299.54 & 299.54 & $\mathrm{NR}^{*}$ & $\mathrm{NR}^{*}$ & 298.16 \\
\hline$Q G$ (Mvar) & 133.9 & 130.83 & 130.94 & $\mathrm{NR}^{*}$ & $\mathrm{NR}^{*}$ & 130.93 \\
\hline Reduction in PLoss (\%) & 0 & 8.4 & 7.4 & 6.6 & 8.3 & 21.19 \\
\hline Total PLoss (Mw) & 17.55 & 16.07 & 16.25 & 16.38 & 16.09 & 13.83 \\
\hline
\end{tabular}

NR* - Not reported.

Then the proposed Enriched Artificial Fish swarm (EAFS) algorithm has been tested, in IEEE 57 Bus system. Table 7 shows the constraints of control variables, Table 8 shows the limits of reactive power generators and comparison results are presented in Table 9.

Table 7. Constraints of control variables

\begin{tabular}{cccc}
\hline System & Variables & Minimum (PU) & Maximum (PU) \\
\hline \multirow{2}{*}{ IEEE 57 Bus } & Generator Voltage & 0.95 & 1.1 \\
& Transformer Tap & 0.9 & 1.1 \\
& VAR Source & 0 & 0.20 \\
\hline
\end{tabular}

Table 8. Constrains of reactive power generators

\begin{tabular}{|c|c|c|c|}
\hline System & Variables & Q Minimum (PU) & Q Maximum (PU) \\
\hline & 1 & -140 & 200 \\
\hline \multirow[t]{6}{*}{ IEEE 57 Bus } & 2 & -17 & 50 \\
\hline & 3 & -10 & 60 \\
\hline & 6 & -8 & 25 \\
\hline & 8 & -140 & 200 \\
\hline & 9 & -3 & 9 \\
\hline & 12 & -150 & 155 \\
\hline
\end{tabular}


Table 9. Simulation results of IEEE-57 system

\begin{tabular}{|c|c|c|c|c|c|c|}
\hline Control variables & Base case & MPSO [19] & PSO [19] & CGA [19] & AGA [19] & EAFS \\
\hline$V G 1$ & 1.040 & 1.093 & 1.083 & 0.968 & 1.027 & 1.029 \\
\hline$V G 2$ & 1.010 & 1.086 & 1.071 & 1.049 & 1.011 & 1.015 \\
\hline$V G 3$ & 0.985 & 1.056 & 1.055 & 1.056 & 1.033 & 1.034 \\
\hline$V G 6$ & 0.980 & 1.038 & 1.036 & 0.987 & 1.001 & 1.012 \\
\hline$V G 8$ & 1.005 & 1.066 & 1.059 & 1.022 & 1.051 & 1.039 \\
\hline$V G 9$ & 0.980 & 1.054 & 1.048 & 0.991 & 1.051 & 1.010 \\
\hline$V G 12$ & 1.015 & 1.054 & 1.046 & 1.004 & 1.057 & 1.042 \\
\hline Tap 19 & 0.970 & 0.975 & 0.987 & 0.920 & 1.030 & 0.953 \\
\hline Tap 20 & 0.978 & 0.982 & 0.983 & 0.920 & 1.020 & 0.935 \\
\hline Tap 31 & 1.043 & 0.975 & 0.981 & 0.970 & 1.060 & 0.927 \\
\hline Tap 35 & 1.000 & 1.025 & 1.003 & $\mathrm{NR}^{*}$ & $\mathrm{NR}^{*}$ & 1.019 \\
\hline Tap 36 & 1.000 & 1.002 & 0.985 & $\mathrm{NR}^{*}$ & $\mathrm{NR}^{*}$ & 1.006 \\
\hline Tap 37 & 1.043 & 1.007 & 1.009 & 0.900 & 0.990 & 1.002 \\
\hline Tap 41 & 0.967 & 0.994 & 1.007 & 0.910 & 1.100 & 0.995 \\
\hline Tap 46 & 0.975 & 1.013 & 1.018 & 1.100 & 0.980 & 1.014 \\
\hline Tap 54 & 0.955 & 0.988 & 0.986 & 0.940 & 1.010 & 0.973 \\
\hline Tap 58 & 0.955 & 0.979 & 0.992 & 0.950 & 1.080 & 0.961 \\
\hline Tap 59 & 0.900 & 0.983 & 0.990 & 1.030 & 0.940 & 0.966 \\
\hline Tap 65 & 0.930 & 1.015 & 0.997 & 1.090 & 0.950 & 1.008 \\
\hline Tap 66 & 0.895 & 0.975 & 0.984 & 0.900 & 1.050 & 0.952 \\
\hline Tap 71 & 0.958 & 1.020 & 0.990 & 0.900 & 0.950 & 1.006 \\
\hline Tap 73 & 0.958 & 1.001 & 0.988 & 1.000 & 1.010 & 1.009 \\
\hline Tap 76 & 0.980 & 0.979 & 0.980 & 0.960 & 0.940 & 0.962 \\
\hline Tap 80 & 0.940 & 1.002 & 1.017 & 1.000 & 1.000 & 1.006 \\
\hline QC 18 & 0.1 & 0.179 & 0.131 & 0.084 & 0.016 & 0.179 \\
\hline$Q C 25$ & 0.059 & 0.176 & 0.144 & 0.008 & 0.015 & 0.161 \\
\hline QC 53 & 0.063 & 0.141 & 0.162 & 0.053 & 0.038 & 0.142 \\
\hline$P G(\mathrm{MW})$ & 1278.6 & 1274.4 & 1274.8 & 1276 & 1275 & 1267.71 \\
\hline$Q G$ (Mvar) & 321.08 & 272.27 & 276.58 & 309.1 & 304.4 & 271.84 \\
\hline Reduction in PLoss (\%) & 0 & 15.4 & 14.1 & 9.2 & 11.6 & 24.55 \\
\hline Total PLoss (Mw) & 27.8 & 23.51 & 23.86 & 25.24 & 24.56 & 20.973 \\
\hline
\end{tabular}

NR* - Not reported.

Then the proposed Enriched Artificial Fish swarm (EAFS) algorithm has been tested, in IEEE 118 Bus system. Table 10 shows the constraints of control variables and comparison results are presented in Table 11.

Table 10. Constraints of control variables

\begin{tabular}{cccc}
\hline System & Variables & Minimum (PU) & Maximum (PU) \\
\hline \multirow{2}{*}{ IEEE 118 Bus } & Generator Voltage & 0.95 & 1.1 \\
& Transformer Tap & 0.9 & 1.1 \\
& VAR Source & 0 & 0.20 \\
\hline
\end{tabular}


Table 11. Simulation results of IEEE-118 system

\begin{tabular}{|c|c|c|c|c|c|c|}
\hline Control variables & Base case & MPSO [19] & PSO [19] & PSO [19] & CLPSO [19] & EAFS \\
\hline$V G 1$ & 0.955 & 1.021 & 1.019 & 1.085 & 1.033 & 1.019 \\
\hline$V G 4$ & 0.998 & 1.044 & 1.038 & 1.042 & 1.055 & 1.048 \\
\hline$V G 6$ & 0.990 & 1.044 & 1.044 & 1.080 & 0.975 & 1.023 \\
\hline$V G 8$ & 1.015 & 1.063 & 1.039 & 0.968 & 0.966 & 1.005 \\
\hline$V G \quad 10$ & 1.050 & 1.084 & 1.040 & 1.075 & 0.981 & 1.014 \\
\hline$V G 12$ & 0.990 & 1.032 & 1.029 & 1.022 & 1.009 & 1.023 \\
\hline$V G \quad 15$ & 0.970 & 1.024 & 1.020 & 1.078 & 0.978 & 1.039 \\
\hline$V G 18$ & 0.973 & 1.042 & 1.016 & 1.049 & 1.079 & 1.042 \\
\hline$V G 19$ & 0.962 & 1.031 & 1.015 & 1.077 & 1.080 & 1.035 \\
\hline$V G \quad 24$ & 0.992 & 1.058 & 1.033 & 1.082 & 1.028 & 1.014 \\
\hline$V G 25$ & 1.050 & 1.064 & 1.059 & 0.956 & 1.030 & 1.033 \\
\hline$V G 26$ & 1.015 & 1.033 & 1.049 & 1.080 & 0.987 & 1.056 \\
\hline$V G 27$ & 0.968 & 1.020 & 1.021 & 1.087 & 1.015 & 0.905 \\
\hline$V G \quad 31$ & 0.967 & 1.023 & 1.012 & 0.960 & 0.961 & 0.904 \\
\hline$V G 32$ & 0.963 & 1.023 & 1.018 & 1.100 & 0.985 & 0.912 \\
\hline$V G \quad 34$ & 0.984 & 1.034 & 1.023 & 0.961 & 1.015 & 1.006 \\
\hline$V G 36$ & 0.980 & 1.035 & 1.014 & 1.036 & 1.084 & 1.005 \\
\hline$V G \quad 40$ & 0.970 & 1.016 & 1.015 & 1.091 & 0.983 & 0.964 \\
\hline$V G 42$ & 0.985 & 1.019 & 1.015 & 0.970 & 1.051 & 1.003 \\
\hline$V G 46$ & 1.005 & 1.010 & 1.017 & 1.039 & 0.975 & 1.010 \\
\hline$V G 49$ & 1.025 & 1.045 & 1.030 & 1.083 & 0.983 & 1.001 \\
\hline$V G 54$ & 0.955 & 1.029 & 1.020 & 0.976 & 0.963 & 0.929 \\
\hline$V G 55$ & 0.952 & 1.031 & 1.017 & 1.010 & 0.971 & 0.962 \\
\hline$V G 56$ & 0.954 & 1.029 & 1.018 & 0.953 & 1.025 & 0.941 \\
\hline$V G 59$ & 0.985 & 1.052 & 1.042 & 0.967 & 1.000 & 0.969 \\
\hline$V G \quad 61$ & 0.995 & 1.042 & 1.029 & 1.093 & 1.077 & 0.972 \\
\hline$V G \quad 62$ & 0.998 & 1.029 & 1.029 & 1.097 & 1.048 & 0.988 \\
\hline$V G \quad 65$ & 1.005 & 1.054 & 1.042 & 1.089 & 0.968 & 1.007 \\
\hline$V G 66$ & 1.050 & 1.056 & 1.054 & 1.086 & 0.964 & 1.003 \\
\hline$V G 69$ & 1.035 & 1.072 & 1.058 & 0.966 & 0.957 & 1.056 \\
\hline$V G 70$ & 0.984 & 1.040 & 1.031 & 1.078 & 0.976 & 1.033 \\
\hline$V G 72$ & 0.980 & 1.039 & 1.039 & 0.950 & 1.024 & 1.029 \\
\hline$V G 73$ & 0.991 & 1.028 & 1.015 & 0.972 & 0.965 & 1.018 \\
\hline$V G 74$ & 0.958 & 1.032 & 1.029 & 0.971 & 1.073 & 1.012 \\
\hline$V G 76$ & 0.943 & 1.005 & 1.021 & 0.960 & 1.030 & 1.010 \\
\hline$V G 77$ & 1.006 & 1.038 & 1.026 & 1.078 & 1.027 & 1.028 \\
\hline$V G 80$ & 1.040 & 1.049 & 1.038 & 1.078 & 0.985 & 1.029 \\
\hline$V G 85$ & 0.985 & 1.024 & 1.024 & 0.956 & 0.983 & 1.012 \\
\hline$V G 87$ & 1.015 & 1.019 & 1.022 & 0.964 & 1.088 & 1.015 \\
\hline$V G 89$ & 1.000 & 1.074 & 1.061 & 0.974 & 0.989 & 1.044 \\
\hline$V G 90$ & 1.005 & 1.045 & 1.032 & 1.024 & 0.990 & 1.033 \\
\hline$V G 91$ & 0.980 & 1.052 & 1.033 & 0.961 & 1.028 & 1.002 \\
\hline
\end{tabular}




\begin{tabular}{|c|c|c|c|c|c|c|}
\hline$V G 92$ & 0.990 & 1.058 & 1.038 & 0.956 & 0.976 & 1.039 \\
\hline$V G 99$ & 1.010 & 1.023 & 1.037 & 0.954 & 1.088 & 1.011 \\
\hline$V G \quad 100$ & 1.017 & 1.049 & 1.037 & 0.958 & 0.961 & 1.022 \\
\hline$V G 103$ & 1.010 & 1.045 & 1.031 & 1.016 & 0.961 & 1.018 \\
\hline$V G 104$ & 0.971 & 1.035 & 1.031 & 1.099 & 1.012 & 1.007 \\
\hline$V G 105$ & 0.965 & 1.043 & 1.029 & 0.969 & 1.068 & 1.056 \\
\hline$V G 107$ & 0.952 & 1.023 & 1.008 & 0.965 & 0.976 & 1.015 \\
\hline$V G 110$ & 0.973 & 1.032 & 1.028 & 1.087 & 1.041 & 1.014 \\
\hline$V G 111$ & 0.980 & 1.035 & 1.039 & 1.037 & 0.979 & 1.003 \\
\hline$V G 112$ & 0.975 & 1.018 & 1.019 & 1.092 & 0.976 & 1.092 \\
\hline$V G 113$ & 0.993 & 1.043 & 1.027 & 1.075 & 0.972 & 1.001 \\
\hline$V G 116$ & 1.005 & 1.011 & 1.031 & 0.959 & 1.033 & 1.006 \\
\hline Tap 8 & 0.985 & 0.999 & 0.994 & 1.011 & 1.004 & 0.935 \\
\hline Tap 32 & 0.960 & 1.017 & 1.013 & 1.090 & 1.060 & 1.004 \\
\hline Tap 36 & 0.960 & 0.994 & 0.997 & 1.003 & 1.000 & 0.950 \\
\hline Tap 51 & 0.935 & 0.998 & 1.000 & 1.000 & 1.000 & 0.932 \\
\hline Tap 93 & 0.960 & 1.000 & 0.997 & 1.008 & 0.992 & 1.019 \\
\hline Tap 95 & 0.985 & 0.995 & 1.020 & 1.032 & 1.007 & 0.978 \\
\hline Tap 102 & 0.935 & 1.024 & 1.004 & 0.944 & 1.061 & 1.028 \\
\hline Tap 107 & 0.935 & 0.989 & 1.008 & 0.906 & 0.930 & 0.942 \\
\hline Tap 127 & 0.935 & 1.010 & 1.009 & 0.967 & 0.957 & 1.013 \\
\hline$Q C 34$ & 0.140 & 0.049 & 0.048 & 0.093 & 0.117 & 0.025 \\
\hline$Q C 44$ & 0.100 & 0.026 & 0.026 & 0.093 & 0.098 & 0.017 \\
\hline$Q C 45$ & 0.100 & 0.196 & 0.197 & 0.086 & 0.094 & 0.169 \\
\hline$Q C 46$ & 0.100 & 0.117 & 0.118 & 0.089 & 0.026 & 0.132 \\
\hline QC 48 & 0.150 & 0.056 & 0.056 & 0.118 & 0.028 & 0.050 \\
\hline QC 74 & 0.120 & 0.120 & 0.120 & 0.046 & 0.005 & 0.143 \\
\hline QC 79 & 0.200 & 0.139 & 0.140 & 0.105 & 0.148 & 0.110 \\
\hline QC 82 & 0.200 & 0.180 & 0.180 & 0.164 & 0.194 & 0.164 \\
\hline$Q C 83$ & 0.100 & 0.166 & 0.166 & 0.096 & 0.069 & 0.135 \\
\hline QC 105 & 0.200 & 0.189 & 0.190 & 0.089 & 0.090 & 0.168 \\
\hline QC 107 & 0.060 & 0.128 & 0.129 & 0.050 & 0.049 & 0.140 \\
\hline QC 110 & 0.060 & 0.014 & 0.014 & 0.055 & 0.022 & 0.010 \\
\hline PG(MW) & 4374.8 & 4359.3 & 4361.4 & $\mathrm{NR}^{*}$ & $\mathrm{NR}^{*}$ & 4381.67 \\
\hline QG(MVAR) & 795.6 & 604.3 & 653.5 & $* \mathrm{NR}^{*}$ & $\mathrm{NR}^{*}$ & 612.92 \\
\hline Reduction in PLOSS(\%) & 0 & 11.7 & 10.1 & 0.6 & 1.3 & 13.53 \\
\hline Total PLOSS (Mw) & 132.8 & 117.19 & 119.34 & 131.99 & 130.96 & 114.83 \\
\hline
\end{tabular}

NR* - Not reported.

Then IEEE 300 bus system [18] is used as test system to validate the performance of the Enriched Artificial Fish swarm (EAFS) algorithm. Table 12 shows the comparison of real power loss obtained after optimization.

Table 12. Comparison of Real Power Loss

\begin{tabular}{ccccc}
\hline Parameter & Method EGA [21] & Method EEA [21] & Method CSA [20] & EAFS \\
\hline PLOSS (MW) & 646.2998 & 650.6027 & 635.8942 & 611.6372 \\
\hline
\end{tabular}




\section{Conclusion}

In this work Enriched Artificial Fish swarm (EAFS) algorithm successfully solved the optimal reactive power problem. In the proposed algorithm which uses the adjustment strategy of exploration range of the artificial fish in iterations. Bulletin board is utilized for recording the optimal position with the utmost food concentration which has been found by fish swarm. In initial iteration, the bulletin board will record the optimal position of the preliminary positions of artificial fish. When the artificial fish moves to a new-fangled position for finding food with superior food concentration, the bulletin board is modernized to record the optimal position discovered so far. Proposed Enriched Artificial Fish swarm (EAFS) algorithm has been tested in standard IEEE 14, 30, 57,118,300 bus test systems and simulation results show the projected algorithm reduced the real power loss comprehensively.

\section{References}

[1] K. Y. Lee. (1984). Fuel-cost minimisation for both real and reactive-power dispatches," Proceedings Generation, Transmission and Distribution Conference, vol/issue: 131(3), pp. 85-93.

[2] N. I. Deeb. (1998). An efficient technique for reactive power dispatch using a revised linear programming approach. Electric Power System Research, vol/issue: 15(2), pp. 121-134.

[3] M. R. Bjelogrlic, M. S. Calovic, B. S. Babic. (1990). Application of Newton's optimal power flow in voltage/reactive power control. IEEE Trans Power System, vol. 5, no. 4, pp. 1447-1454.

[4] S. Granville. (1994). Optimal reactive dispatch through interior point methods. IEEE Transactions on Power System, vol/issue: 9(1), pp. 136-146.

[5] N. Grudinin. (1998). Reactive power optimization using successive quadratic programming method. IEEE Transactions on Power System, vol/issue: 13(4), pp. 1219-1225.

[6] Ng Shin Mei, R., Sulaiman, M.H., Mustaffa, Z., Daniyal, H. (2017). Optimal reactive power dispatch solution by loss minimization using moth-flame optimization technique. Appl. Soft Comput. 2017, 59, 210-222.

[7] Chen, G., Liu, L., Zhang, Z., Huang, S. (2017). Optimal reactive power dispatch by improved GSA-based algorithm with the novel strategies to handle constraints. Appl. Soft Comput. 2017, 50, 58-70.

[8] Naderi, E., Narimani, H., Fathi, M., Narimani, M.R. (2017). A novel fuzzy adaptive configuration of particle swarm optimization to solve large-scale optimal reactive power dispatch. Appl. Soft Comput. 2017, 53, 441-456.

[9] Heidari, A.A., Ali Abbaspour, R., Rezaee Jordehi. (2017). A. Gaussian bare-bones water cycle algorithm for optimal reactive power dispatch in electrical power systems. Appl. Soft Comput. 2017, 57, 657-671.

[10] Mahaletchumi Morgan, Nor Rul Hasma Abdullah, Mohd Herwan Sulaiman, Mahfuzah Mustafa, Rosdiyana Samad. (2016). Benchmark Studies on Optimal Reactive Power Dispatch (ORPD) Based Multi-objective Evolutionary Programming (MOEP) Using Mutation Based on Adaptive Mutation Adapter (AMO) and Polynomial Mutation Operator (PMO). Journal of Electrical Systems, 12-1.

[11] Rebecca Ng Shin Mei, Mohd Herwan Sulaiman, Zuriani Mustaffa. (2016). Ant Lion Optimizer for Optimal Reactive Power Dispatch Solution. Journal of Electrical Systems, "Special Issue AMPE2015”, pp. 68-74.

[12] Ram Kishan Mahate, \& Himmat Singh. (2019). Multi-Objective Optimal Reactive Power Dispatch Using Differential Evolution. International Journal of Engineering Technologies and Management Research, 6(2), 27-38.

[13] Gagliano A., Nocera F. (2017). Analysis of the performances of electric energy storage in residential applications. International Journal of Heat and Technology. Vol. 35, Special Issue 1, pp. S41-S48.

[14] Caldera M., Ungaro P., Cammarata G., Puglisi G. (2018). Survey-based analysis of the electrical energy demand in Italian households. Mathematical Modelling of Engineering Problems, Vol. 5, No. 3, pp. 217-224.

[15] M. Basu. (2016). Quasi-oppositional differential evolution for optimal reactive power dispatch. Electrical Power and Energy Systems, vol. 78, pp. 29-40.

[16] T. Weise. (2009). Global Optimization Algorithms - Theory and Application, Germany: it-weise.de (self-published), [Online]. Available: http://www.it-weise.de/.

[17] M.A.K. Azad, A.M.A.C. Rocha, E.M.G.P. Fernandes. (2014). A simplified binary artificial fish swarm algorithm for 0-1 quadratic knapsack problems. Journal of Computation Applied Mathematics, 259, 897-904.

[18] IEEE. (1993). “The IEEE-test systems”. http://www.ee.washington.edu/trsearch/pstca/.

[19] Ali Nasser Hussain, Ali Abdulabbas Abdullah and Omar Muhammed Neda. (2018). Modified Particle Swarm Optimization for Solution of Reactive Power Dispatch. Research Journal of Applied Sciences, Engineering and Technology 15(8): 316-327. 
[20] S. Surender Reddy. (2017). Optimal Reactive Power Scheduling Using Cuckoo Search Algorithm. International Journal of Electrical and Computer Engineering. Vol. 7, No. 5, pp. 2349-2356.

[21] S.S. Reddy. (2014). Faster evolutionary algorithm based optimal power flow using incremental variables. Electrical Power and Energy Systems, vol. 54, pp. 198-210. 